\section{Ergänzung zu Moskitonetzen und Insektiziden Impfung halbiert Malaria-Risiko}

- Der erste aussichtsreiche Malaria-Impfstoff wird seit zwei Jahren in einer großen Phase-III-Studie in sieben afrikanischen Ländern an fast 16000 Kindern geprüft. Einer ersten Zwischenauswertung von 6000 Studienteilnehmern zufolge verspricht der Impfstoff einen Schutz von ca. 50\%.

Die dreimalige Impfung mit der Malaria-Vakzine RTS,S reduzierte bei Kindern im Alter von fünf bis 17 Monaten das Risiko, innerhalb des nächsten Jahres an Malaria zu erkranken, um 56\%. Die Zahl schwerer Malaria-Episoden ging um $47 \%$ zurück. In einer weiteren Analyse von 15460 Säuglingen und Kindern im Alter von sechs Wochen bis 17 Monaten ging die Häufigkeit schwerer Malaria-Episoden um 35\% zurück (N Engl J Med. 2011; published online am 18. Oktober 2011; doi: 10.1056/NEJMoa1102287). Ob auch Todesfälle durch die Malaria-Impfung verhindert werden können, geht aus dieser Auswertung nicht hervor.

\section{Bisher Erfahrungen über ein Jahr}

Die bisherigen Ergebnisse umfassen Beobachtungszeiträume von einem Jahr nach der letzten Impfung. Daten über die langfristige Wirksamkeit sollen Ende 2014 vorliegen. Auch zum Sicherheitsprofil sollen in den nächsten drei Jahren weitere Daten veröffentlicht werden. Bisherige
Analysen der Verträglichkeit ergaben ein gehäuftes Auftreten von fiebrigen Reaktionen.

Der leitende Prüfarzt der Studie, Dr. Tsiri Agbenyega von der Malaria Research Unit am Komfo-Anokye Hospital in Kumasi (Ghana), hält die Ergebnisse für bemerkenswert, da es bisher keine erfolgreiche Impfung gegen Malaria gibt. Auch andere Experten sprechen von einem Erfolg, obwohl die Impfung Kinder nur zu etwa 50\% vor Malaria schützte. Von Ausrottung der Malaria wird also so schnell nicht die Rede sein, und auf zusätzliche Prophylaxemaßnahmen wie Moskitonetze und Insektizide kann weiterhin nicht verzichtet werden sie kamen auch in der Studie in großem Umfang zum Einsatz.

\section{Günstiger Preis in Aussicht}

RTS,S richtet sich gegen ein Oberflächenprotein der Sporozoiten des Malaria-Erregers Plasmodium falciparum. Die Impfung soll die Sporozoiten abfangen, damit sie nicht in die Leber eindringen. Normalerweise vermehren sich die Erreger in der Leber, gelangen von dort in die Blutbahn und infizieren die Erythrozyten, was letztendlich zum Ausbruch der Erkrankung führt und die gefürchteten Symptome hervorruft.

Noch ist der Impfstoff nicht zugelassen. Wenn es soweit ist, soll RTS, $\mathrm{S}$ zu einem

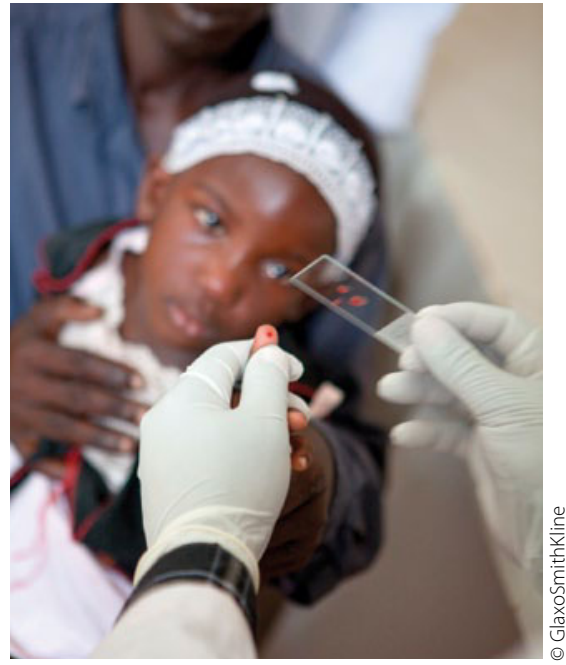

Ein Kind wird in einer Klinik in Bagamoyo auf Malaria getestet.

möglichst niedrigen Preis zur Verfügung gestellt werden, erklärte Andrew Witty, Vorstandsvorsitzender von GlaxoSmithKline. Man wolle lediglich die Herstellungskosten decken und einen kleinen Gewinn von $5 \%$ erzielen, der in weitere Forschungsaktivitäten auf dem Gebiet der Malaria-Impfung investiert werden soll. GSK entwickelt den Impfstoff in Zusammenarbeit mit der PATH Malaria Vaccine Initiative (MVI). Gefördert wird die Entwicklung des Impfstoffs von der Bill \& Melinda Gates Foundation.

- Dr. Judith Neumaie

Quelle: Malaria Vaccine Press Briefing, Telefonkonferenz, Oktober 2011 (Veranstalter: GlaxoSmithKline)

\title{
Herzinsuffizienz \\ Herzfrequenz senken bessert die Prognose
}

— Eine erhöhte Herzfrequenz über 70 Schläge pro Minute ist ein Risikofaktor für kardiovaskuläre Ereignisse für Patienten mit Herzschwäche. Als Mittel zur Herzfrequenzsenkung geeignet sind Betablocker, der $I_{f}$-Kanal-Blocker Ivabradin oder eine Kombination dieser Substanzen. Der Kardiologe Prof. Carsten Tschöpe, Charité Universitätsmedizin in Berlin, erinnerte an die doppelblinde SHIFT-Studie von Karl Swedberg (The Lancet, 2010, Vol. 376, 875-885). 6557 Patienten mit symptoma- tischer Herzinsuffizienz (EF unter 35) mit Sinusrhythmus und einer Frequenz von über 70/Minute wurden 23 Monate lang mit Placebo oder Ivabradin $(2 \times 7,5 \mathrm{mg} / \mathrm{d})$ behandelt. Das Hauptergebnis war, dass das Risiko für einen kardiovaskulären Tod oder Krankenhauseinweisung wegen Verschlechterung der Herzinsuffizienz signifikant gesenkt wurde (793 (24\%) vs. 937 (29\%) Patienten, HR 18\%, p < 0,0001). V. a. Einweisungen und Tod wegen Herzinsuffizienz wurden reduziert. 26 Patienten muss- ten behandelt werden, um ein Ereignis zu verhindern.

Auch in den großen HerzinsuffizienzStudien der 90er-Jahre mit Bisoprolol, Carvedilol und Metoprolol konnte ein günstiger Einfluss der Herzfrequenzsenkung auf die Gesamtmortalität nachgewiesen werden.

\footnotetext{
- Rainer Klawki

Quelle: Symposium der Firma Servier auf der DGK-Herbsttagung in Düsseldorf, Oktober 2011
} 